\title{
On the design of clustered planar phased arrays for wireless power transmission
}

\author{
Toshifumi Moriyama ${ }^{1 a)}$, Lorenzo Poli ${ }^{2}$, and Paolo Rocca ${ }^{2 b)}$ \\ ${ }^{1}$ Graduate School of Engineering, Nagasaki University, \\ 1-14 Bunkyo-machi, Nagasaki 852-8521, Japan \\ ${ }^{2}$ ELEDIA Research Center@ DISI, University of Trento,
}

Via Sommarive, 38123 Trento, Italy

a)t-moriya@nagasaki-u.ac.jp

b) paolo.rocca@disi.unitn.it

\begin{abstract}
In the framework of wireless power transmission applications, the design of planar phased arrays providing maximum beam collection efficiency (i.e., maximizing the ratio between the power radiated over a target area and the total power transmitted by the array) through a subarrayed architecture is addressed. The degrees-of-freedom of the synthesis problem at hand (i.e., the membership of the array elements to clusters and the sub-array amplitudes) are determined by means of an excitation matching technique exploiting the knowledge of the set of independent and optimal weights providing maximum transfer efficiency. A set of representative results is reported to assess the reliability and effectiveness of the approach in yielding beam efficiency values close to the optimal ones with a limited number of sub-arrays.
\end{abstract}

Keywords: antenna arrays, sub-arraying, wireless power transmission Classification: Electromagnetic theory

\section{References}

[1] K. Wu, D. Choudhury and H. Matsumoto: Proc. IEEE 101 (2013) 1271. DOI:10.1109/JPROC.2013.2257590

[2] P. E. Glaser: Proc. IEEE 65 (1977) 1162. DOI:10.1109/PROC.1977.10662

[3] P. E. Glaser: IEEE Trans. Microw. Theory Techn. 40 (1992) 1230. DOI:10. $1109 / 22.141356$

[4] H. Matsumoto: IEEE Microw. Mag. 3 (2002) 36. DOI:10.1109/MMW.2002. 1145674

[5] A. Massa, G. Oliveri, F. Viani and P. Rocca: Proc. IEEE 101 (2013) 1464. DOI:10.1109/JPROC.2013.2245491

[6] G. Oliveri, L. Poli and A. Massa: IEEE Trans. Antennas Propag. 61 (2013) 2490. DOI:10.1109/TAP.2013.2241714

[7] S. Caorsi, A. Massa, M. Pastorino and A. Randazzo: IEEE Trans. Antennas Propag. 53 (2005) 372. DOI:10.1109/TAP.2004.838788

[8] Y. Chen, S. Yang and Z. Nie: IEEE Trans. Antennas Propag. 56 (2008) 1919. DOI:10.1109/TAP.2008.924713

[9] M. D’Urso, T. Isernia and E. F. Meliadò: IEEE Trans. Antennas Propag. 55 (2007) 1059. DOI:10.1109/TAP.2007.893374 
[10] P. Rocca, L. Manica, R. Azaro and A. Massa: IEEE Trans. Antennas Propag. 57 (2009) 280. DOI:10.1109/TAP.2008.2009776

[11] P. Rocca, L. Manica and A. Massa: IEEE Trans. Antennas Propag. 57 (2009) 1684. DOI:10.1109/TAP.2009.2019914

[12] L. Manica, P. Rocca, A. Martini and A. Massa: IEEE Trans. Antennas Propag. 56 (2008) 58. DOI:10.1109/TAP.2007.913037

[13] L. Manica, P. Rocca, M. Benedetti and A. Massa: IEEE Trans. Antennas Propag. 57 (2009) 652. DOI:10.1109/TAP.2009.2013423

[14] W. D. Fisher: J. Am. Statist. Assoc. 53 (1958) 789. DOI:10.1080/01621459. 1958.10501479

\section{Introduction}

Remote powering is another key function enabled by wireless systems along with communication and sensing. Unlike these latter, the concept of wireless power transmission (WPT) is not diffused and still unclear [1]. In WPT, the objective is the transfer of energy between a transmitting and a receiving device without the need of wires or physical connections. Towards this end, near-field coupling of resonant circuits or transmitting antennas and rectifying antennas (i.e., rectennas) can be profitably used when short/medium range or long (electrical) distances are at hand. As for long range WPT, a very challenging and fascinating application is the so-called Space-Base Solar Power (SBSP) aimed at guaranteeing a continuous (i.e., twenty-four hours per day) feeding of the Earth with renewable and clean energy $[2,3,4]$. In short, the idea is to have satellites equipped with extended solar panels that collect solar energy in the space to be converted and delivered to the ground through microwave beams. Then, receiving rectennas convert the RF energy to DC for a successive delivery through the terrestrial power transmission grid. Of course, SBSP is not the only application of long range WPT, but there are many other applicative examples of closer practical interest such as the powering or remote or inaccessible areas in case of natural disasters for civil protection purposes or the wireless charging of electrical vehicles or mobile devices. Accordingly, strategies for the design of highly efficient and affordable antennas systems are needed. The transmitting antenna has to focus the energy in a narrow angular sector to avoid undesired interferences with other wireless systems and unsafe interactions with living beings. To fit those application-driven requirements, antenna arrays with large apertures should be used and the array excitations set to maximize the ratio between the power transmitted in the receiving area and the total radiated power [5]. Theoretically, such a problem has been recently solved in [6] where the excitations of an array of independent elements (i.e., each one equipped with a complete - amplifier and phase shifter - control point), which maximize the beam collection efficiency (BCE), have been analytically determined by solving a generalized eigenvalue problem. Despite the optimality of the arising transmission efficiency, the synthesized array turns out being too complex for practical implementations, bulky, and expensive due to the use of a single-antenna control strategy.

In this letter, a method for designing phased arrays providing maximum $\mathrm{BCE}$, but characterized by simplified array architectures is presented. The array layout is 
organized in clusters, each one containing several radiating elements, and the control of the excitation amplitudes is carried out at the sub-array level. To properly address such a synthesis problem by defining the layout of the sub-array configuration and the values of the sub-array weights, several approaches based on either global optimization algorithms [7, 8], hybrid [9, 10, 11], or ad-hoc deterministic $[12,13]$ techniques have been proposed. Thanks to its efficiency, the Contiguous Partition Method (CPM) presented in [13] is here properly customized to the design of clustered planar phased arrays providing maximum BCE for WPT applications. In such a framework, the key-advantages of the CPM are twofold: (i) it can fully exploit the knowledge of the optimal weights coming from the theoretical approach in [6] being an excitation matching method; (ii) it enables the synthesis of large arrays, as requested in long range WPT, because of its numerical/computational efficiency in dealing with high-dimension problems.

\section{Clustered planar phased array design for WPT}

Let us consider a planar array of $N$ elements located on the $x y$-plane whose array factor is defined as

$$
A F(u, v)=\sum_{n=1}^{N} \sum_{q=1}^{Q} \delta_{c_{n} q} I_{q} \exp \left\{j\left[k\left(u x_{n}+v y_{n}\right)+\varphi_{n}\right]\right\}
$$

where $\left\{I_{q} ; q=1, \ldots, Q\right\}$ is the set of sub-arrayed amplitude coefficients and $\varphi_{n}$, $n=1, \ldots, N$ are the phase weights, one for each element of the array and used for beam steering purposes. Moreover, $u=\sin \theta \cos \phi$ and $v=\sin \theta \sin \phi$ are the angular cosine directions, $\left(x_{x}, y_{n}\right), n=1, \ldots, N$ the coordinates of the array elements, $k=2 \pi / \lambda, \lambda$ being the free space wave-length, and $\delta_{c_{n} q}$ the Kronecker delta equal to $\delta_{c_{n} q}=1$ when $c_{n}=q$ and $\delta_{c_{n} q}=0$, otherwise. The integer values $\left\{c_{n} \in[1, Q] ; n=1, \ldots, N\right\}$ identify the membership of each $n$-th array element to a $q$-th cluster $(1 \leq q \leq Q)[10,11,12,13]$.

The degrees-of-freedom of the synthesis problem are the values of the sub-array amplitude coefficients $\left\{I_{q} ; q=1, \ldots, Q\right\}$ and the clustering of the array elements $\left\{c_{n} ; n=1, \ldots, N\right\}$. In order to define these unknowns, the synthesis problem is cast as the solution of an excitation matching problem in which the best least-square and stepwise approximation [14] of the set of independent and optimal coefficients $\left\{I_{n}^{B C E} ; n=1, \ldots, N\right\}$ maximizing the desired performance metric (i.e., the BCE) is addressed through the approach proposed in [13].

Hence, the set $\left\{I_{n}^{B C E} ; n=1, \ldots, N\right\}$ is firstly obtained through the maximization of the BCE [6]

$$
B C E=\frac{\iint_{\Omega}|A F(u, v)|^{2} d u d v}{\iint|A F(u, v)|^{2} d u d v}
$$

$\Omega$ being the angular sector where it is intended to concentrate the maximum amount of transmitted power, according to the method proposed in [6]. It is important to observe that the reference excitations $\left\{I_{n}^{B C E} ; n=1, \ldots, N\right\}$ are determined for the broadside case [i.e., when the peak of the main lobe is directed at $(u, v)=(0,0)$ ] by imposing $\varphi_{n}=0, n=1, \ldots, N$, namely using only real amplitude weights. 
The problem unknowns, $\left\{I_{q} ; q=1, \ldots, Q\right\}$ and $\left\{c_{n} ; n=1, \ldots, N\right\}$, are then determined with the CPM [13] through the minimization of the following cost function [14]

$$
\Psi\left(c_{n}\right)=\frac{1}{N} \sum_{n=1}^{N}\left|I_{n}^{B C E}-\sum_{q=1}^{Q} \delta_{c_{n} q} I_{q}\left(c_{n}\right)\right|^{2}
$$

aimed at quantifying the mean square distance between the optimal coefficients $\left\{I_{n}^{B C E} ; n=1, \ldots, N\right\}$ and the stepwise approximation $\left\{\sum_{q=1}^{Q} \delta_{c_{n} q} I_{q}\left(c_{n}\right) ; n=1, \ldots, N\right\}$ where the $Q$ sub-array amplitude weights of each trial clustering are given by $[13,14]$

$$
I_{q}\left(c_{n}\right)=\frac{\sum_{n=1}^{N} \sum_{q=1}^{Q} \delta_{c_{n} q} I_{n}^{B C E}}{\sum_{n=1}^{N} \sum_{q=1}^{Q} \delta_{c_{n} q}}, \quad q=1, \ldots, Q
$$

The search of the sub-array configuration, $\left\{c_{n} ; n=1, \ldots, N\right\}$, minimizing (3) is carried out according to the graph-based procedure described in [13] where the initial guess sub-array configuration is iteratively updated by changing the subarray membership of a particular subset of array elements, called border elements, to allow a quick and efficient sampling of the solution space.

\section{Numerical results}

To investigate the effectiveness of the CPM-based WPT design method, a square planar array with $N=400$ elements uniformly-spaced by $d=\lambda / 2$ along the $x$ and $y$ coordinates has been taken into account. As for the optimal array coefficients maximizing the $\mathrm{BCE}$, they have been generated according to [6] by setting the target area $\Omega$ as follows: $-0.2 \leq u \leq 0.2$ and $-0.2 \leq v \leq 0.2$. By using independent excitations, the optimal array radiates the (reference) power pattern in Fig. 1 with a beam efficiency equal to $B C E^{\text {opt }}=0.99$.

Successively, two different clustered phased arrays $(Q=2$ and $Q=4)$ have been synthesized with the CPM-based method. The arising clustering arrange-

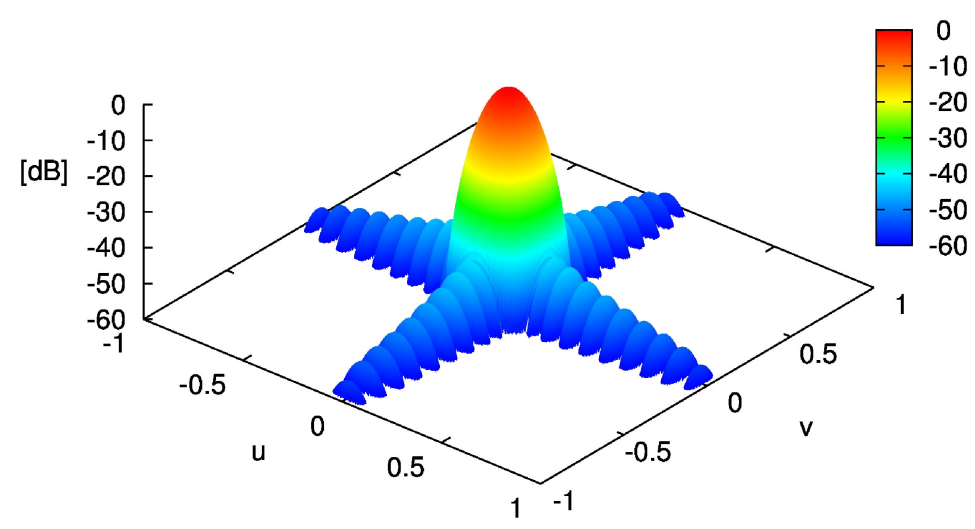

Fig. 1. Reference power pattern providing optimal BCE value [6]. 


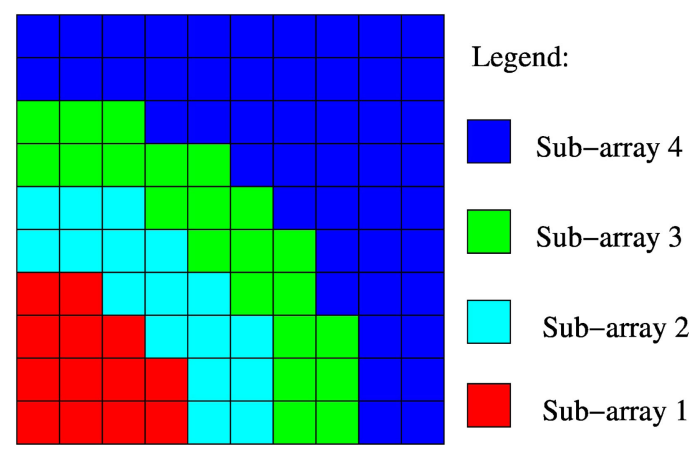

(a)

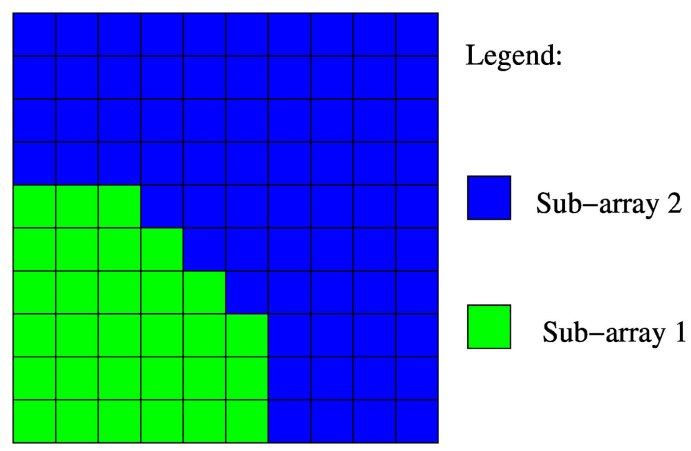

(b)

Fig. 2. CPM-synthesized excitation coefficients when (a) $Q=4$ and (b) $Q=2$.

ments, $\left\{c_{n} ; n=1, \ldots, N\right\}$, and the corresponding sub-array amplitude coefficients, $\left\{I_{q} ; q=1, \ldots, Q\right\}$, obtained in less than 2 [sec] on a standard laptop PC, are given in Fig. 2 and in Table I, respectively, while the radiated patterns are shown in Fig. 3 .

As it can be observed, only one quadrant of the total array layout is reported in Fig. 2 because of the quadrantal symmetry of the arising antenna array. Concerning the BCE, it is worth pointing out that the CPM-based synthesis is able to guarantee performances very close to the optimal one $\left[B C E^{Q=4}=0.98\right.$ vs. $B C E^{o p t}=0.99$ Fig. 3(a)] with a drastic simplification of the array beam-forming network (BFN) from $N / 4=100$ of the optimal solution in [6] down to $Q=4$ independent control points. As expected, further reducing the BFN complexity (i.e., $Q=2$ ) causes an increase of the mismatch with the reference solution [Fig. 3(b) vs. Fig. 1] also in terms of $\mathrm{BCE}$ even though smaller than $8 \%\left(B C E^{Q=2}=0.91\right)$.

Table I. Sub-array amplitude weights.

\begin{tabular}{|c|c|c|}
\hline & $Q=2$ & $Q=4$ \\
\hline$q$ & \multicolumn{2}{|c|}{$I_{q}$} \\
\hline 1 & 0.648 & 0.831 \\
\hline 2 & 0.125 & 0.509 \\
\hline 3 & - & 0.255 \\
\hline 4 & - & 0.064 \\
\hline
\end{tabular}




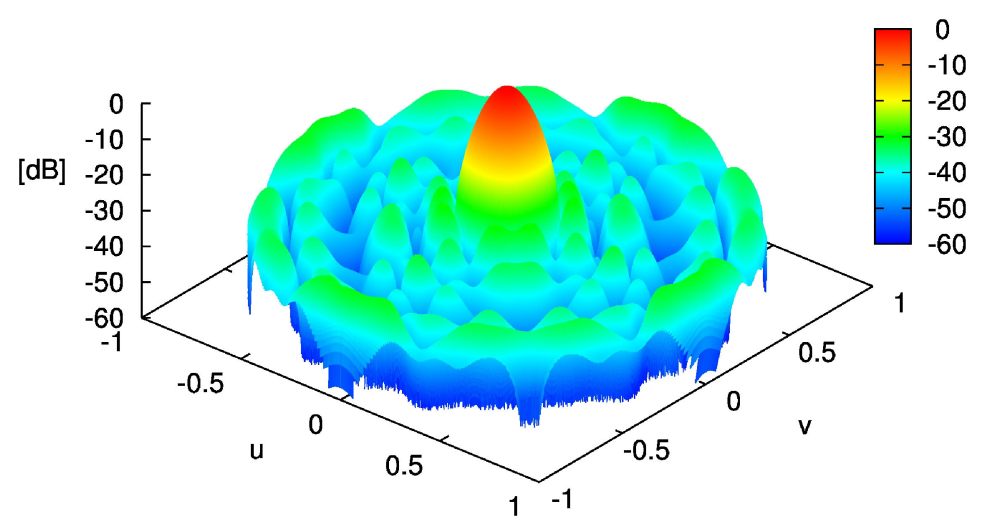

(a)

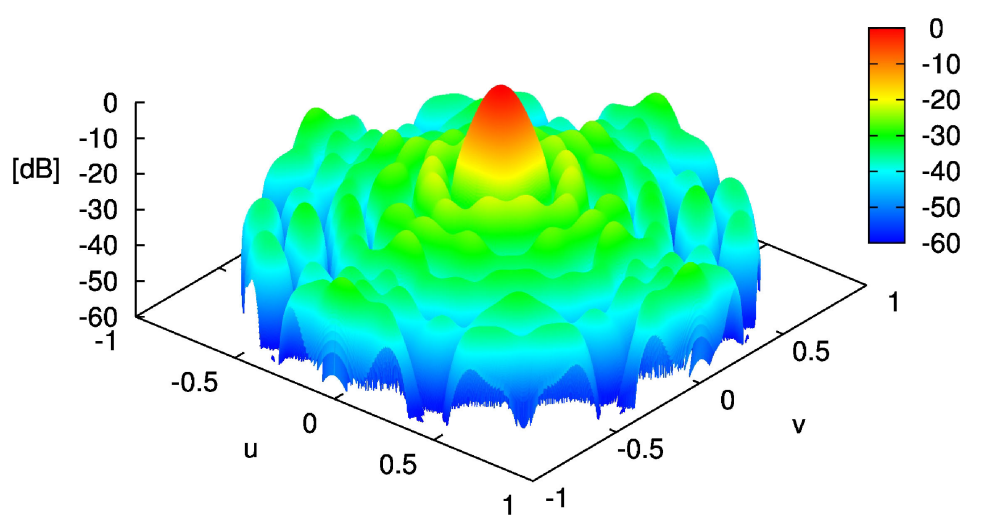

(b)

Fig. 3. CPM-synthesized compromise power pattern when (a) $Q=4$ and (b) $Q=2$.

\section{Conclusions}

The design of clustered planar phased arrays with maximum beam collection efficiency to be adopted as transmitting antennas in WPT systems has been addressed. The availability of reference and independent coefficients providing the optimal BCE values for a given target area where to transfer the maximum energy has been exploited thanks to a customized CPM-based approach able to determine in a quick and reliable way, also in high-dimension problems, the clustered array-elements configurations and the corresponding sub-array amplitude excitations. Representative results proved the effectiveness and the efficiency of the proposed method also in dealing with large phased array designs where high beam efficiency values have been obtained with a limited number of clusters. 